\title{
Different distribution of breast ductal carcinoma in situ, ductal carcinoma in situ with microinvasion, and invasion breast cancer
}

Zhang Wei ${ }^{1}$, Gao Er-li', Zhou Yi-li', Zhai Qi ${ }^{1}$, Zou Zhang-yong ${ }^{1}$, Guo Gui-long ${ }^{1 *}$, Chen Guo-rong ${ }^{2}$, Zheng Hua-min ${ }^{3}$, Huang Guan-li ${ }^{1}$ and Zhang Xiao-hua ${ }^{1}$

\begin{abstract}
Background: Breast ductal cancer in situ (DCIS) can recur or progress to invasive ductal cancer (IDC), and the interim stage include DCIS with microinvasion (DCIS-Mi). In this article, we attempt to study the study the differences of clinicopathological features, imaging data, and immunohistochemical-based subtypes among DCIS, DCIS-Mi, and IDC.

Methods: In this retrospective study, we attempt to compare the clinicopathological features, immunohistochemical results and imaging data of 866 patients (included 73 DCIS, 72 DCIS-Mi, and 721 IDC).

Results: Patients with DCIS and DCIS-Mi were younger than those with IDC $(P=0.007)$. DCIS and DCIS-Mi often happened in premenopausal women while IDC was opposite $(P<0.001)$. The incidence of IDC with node-positive was significantly higher than it in DCIS and DCIS-Mi $(P<0.001)$. We also observed that the Her2-positive was more often found in patients with pure DCIS compared to those with DCIS-Mi and DCIS-I $(P<0.001)$. There was a significant difference between the four subgroups (Luminal-A, Luminal-B, ERBB2+, Basal-like) from DCIS, DCIS-Mi, and IDC ( $P$ <0.001). Basal-like patients were fewer than other subgroups in DCIS, DCIS-Mi, and IDC. The incidence of the first performance of ultrasound (catheter winded and nodular mass) and mammography (nodular mass) had significantly difference among patients with DCIS, DCIS-Mi, and IDC $(P<0.001)$.
\end{abstract}

Conclusions: Different clinicopathological, immunohistochemical, and imaging features among DCIS, DCIS-Mi, and IDC indicate that they are distinct entities. A larger sample size is needed for further study.

Keywords: Breast neoplasms, Ductal carcinoma in situ, Ductal carcinoma in situ with microinvasion, Invasion breast cancer

\section{Background}

Breast cancer is one of the most common malignant tumors for women [1]. In recent years, the incidence of breast cancer shows an increasing trend in China. Breast ductal cancer in situ (DCIS) is a neoplastic proliferation of epithelial cells confined to the ductal-lobular system without tumor invasion through the basement membrane [2]. Due to the extensively use of mammographic imaging, the number of patients with DCIS and DCIS with microinvasive (DCIS-Mi) is increasing. According

\footnotetext{
* Correspondence: oncologygg|@163.com

'Department of Oncology, The First Affiliated Hospital of Wenzhou Medical College, Wenzhou, Zhejiang, People's Republic of China

Full list of author information is available at the end of the article
}

to the criteria of the American Joint Committee on Cancer (AJCC), DCIS-Mi is defined as DCIS with a microscopic focus of invasion $\leq 1 \mathrm{~mm}$ in the longest diameter, which accounts for approximately $10 \%$ to $20 \%$ of DCIS cases [3,4]. DCIS-Mi included the dominant lesion, which is in-situ carcinoma and one or more foci of infiltration [5-8]. And international scholars consider that it may be the interim stage in the progression from DCIS to invasive breast cancer (IDC) $[9,10]$. Recent studies revealed that DCIS-Mi was potential for invasion and metastasis differentiated form pure DCIS, which also result for the different surgical strategy [11,12]. So DCISMi may represent a distinct entity.

\section{Biomed Central}

(c) 2012 Wei et al.; licensee BioMed Central Ltd. This is an Open Access article distributed under the terms of the Creative Commons Attribution License (http://creativecommons.org/licenses/by/2.0), which permits unrestricted use, distribution, and reproduction in any medium, provided the original work is properly cited. 
Today a multistep model of human breast cancer progression hypothesizes that IDC develops through sequential stages, from premalignant hyperplastic breast lesions with or without atypia to carcinoma in situ to invasive carcinoma [2,13-15]. Very few studies have paid attention to the association of clinicopathological and immunohistochemical (IHC) features among DCIS, DCIS-Mi, and IDC. So we studied the differences of clinicopathological features as well as IHC marker-based subtypes and imaging data among DCIS, DCIS-Mi, and IDC.

\section{Methods \\ Patients}

We retrospectively collected 953 breast cancer patients from February 2006 to April 2010. Among the 953 patients, 96 cases were excluded because their first tumor lumpectomies were not in our hospital and there were no data of specimens. Finally, 73 patients with DCIS (9.29\%), 72 patients with DCIS-Mi (9.16\%), and 721 patients with IDC (83.26\%) were enrolled in this research. All the patients accepted physical examination, chest radioscopy, mammography, ECG, complete blood count, routine biochemical tests, and ultrasound (US) (included breasts, axillary fossa, abdomen, and pelvis). Each patient was treated with surgery by either lumpectomy or mastectomy, and whether doing axillary lymph node dissection was followed by adjuvant therapies according to the standard guideline. All patients were treated with lumpectomy or mastectomy (most with axillary lymph node dissection) followed by adjuvant therapies according to the standard guideline.

The age of the 866 patients ranged from 24 to 87 years old (the median age at diagnosis was 52 years old). Median follow-up of those patients was 35 months (range, 1 to 118 months). We collected age, menopausal status, tumor size, lymph node status, status of IHC (ER, PR, and HER2), family history, and the history of breast surgery. The pathologic and IHC outcomes were checked by our senior pathologists in our hospital. This study was approved by the Institutional Review Board of Wenzhou Medicine University. Written informed consents were obtained from the patients for publication of this report.

\section{Pathologic definition}

DCIS is defined as clonal proliferation of cells growing within the basement membrane-bound structures of the breast [16]. DCIS-Mi is defined as DCIS with extension of cancer cells beyond the basement membrane and invasion $\leq 1 \mathrm{~mm}$ in the longest diameter. Those tumor cells which are invasive breast ductal basement membrane-bound structure were identified as IDC. The specimens of all patients in our study were diagnosed by three board-certified pathologists with a mean 11 years (17, 9, and 7 years, respectively) of experience who were not aware of the result of pathological examination from the former pathologist.

\section{Immunohistochemistry and scoring}

The status of ER, PR, and HER2 confirmed by IHC staining, which was performed through a standard operating procedure in our Department of Pathology. ER and PR expression in the nuclei of tumor cells, Her-2 expression in the cytoplasm, positive cell is that cells contain brown grains. A score of 0 required no staining seen, 1 required $\leq 25 \%$ of cells positive, 2 required $25 \%$ to $50 \%$ of cells stained, 3 required $50 \%$ to $75 \%$ of positive cells, and 4 required $>75 \%$ of staining cells.

\section{Statistical analysis}

We compared clinicopathological, IHC, and imaging characteristics in the patient with DCIS, DCIS-Mi, and IDC. For each group we estimated from the date of the surgery by the life table. Tests of association and correlation were conducted by using the $n \times n$ Pearson's $x^{2}$ test (or Fisher's exact test when appropriate). One-way ANOVA was used to compare continuous variables among two or more groups. $P<0.05$ was considered statistically significant. Statistical analyses were performed using SPSS software version 17.0.

\section{Results}

The clinicopathological characteristics of patients are shown in Table 1. Patients with DCIS and DCIS-Mi were significantly younger than those with IDC $(P=0.007)$. Patients with DCIS and DCIS-Mi were more often found to have the status of premenopausal when compare to those with IDC $(P \leq 0.001)$. The incidence of lymph node metastasis from patients with IDC (38.75\%) was significantly higher compared to the patients with pure DCIS (6.85\%) and patients with DCIS-Mi (20.83\%) $(P \leq 0.001)$. We also observed that the number of status of Her2 expressed positive in patients with DCIS and DCIS-Mi was higher than in those with IDC $(P<0.001)$ but we found that the tumor size and family history of breast cancer in a first-degree relative seemed to have no difference in the three groups $(P>0.05)$. We divided patients into four subgroups: Luminal-A, Luminal-B, ERBB2+, and Basal-like. We found no significant difference between the four subgroups regarding DCIS, DCIS-Mi, and IDC.

Table 2 showed the clinical data of the first clinical performances and diagnostic methods among DCIS, DCIS-Mi, and IDC groups. For the first clinical performance for diagnosis, palpable mass was the main symptom with no significant difference in the three groups $(P=0.52)$. The incidence of nipple discharge was similar in the 
Table 1 Characteristics of the three patient populations: DCIS, DCIS-Mi, and IDC

\begin{tabular}{|c|c|c|c|c|}
\hline Parameter & DCIS & DCIS-Mi & IDC & $P$ value \\
\hline Total number & 73 & 72 & 721 & \\
\hline Age (mean \pm SD) (year) & $48.96 \pm 1.13$ & $49.34 \pm 1.01$ & $52.23 \pm 0.43$ & 0.007 \\
\hline Menopausal & & & & $<0.001$ \\
\hline Postmenopausal & $21(28.77)$ & $28(38.89)$ & $415(57.60)$ & \\
\hline Premenopausal & $52(71.23)$ & $44(61.11)$ & $306(42.40)$ & \\
\hline Family history & & & & 0.791 \\
\hline Positive & $6(8.2)$ & $6(8.3)$ & $48(6.7)$ & \\
\hline Negative & $67(91.8)$ & $66(91.7)$ & $673(93.3)$ & \\
\hline Tumor size $(\mathrm{cm})$ & & & & 0.238 \\
\hline$<2$ & $38(52.05)$ & $32(44.44)$ & $302(41.9)$ & \\
\hline$\geq 2$ & $35(47.95)$ & $40(55.56)$ & $419(58.1)$ & \\
\hline Lymph node & & & & 0.000 \\
\hline Positive & $5(6.85)$ & $15(20.83)$ & 279 (38.75) & \\
\hline Negative & $68(93.15)$ & $57(79.17)$ & $442(61.25)$ & \\
\hline$E R$ & & & & 0.265 \\
\hline Positive & $44(60.27)$ & $42(58.33)$ & $478(66.25)$ & \\
\hline Negative & $29(39.73)$ & $30(41.67)$ & $243(33.75)$ & \\
\hline$P R$ & & & & 0.736 \\
\hline Positive & $41(56.20)$ & $37(51.40)$ & $405(56.25)$ & \\
\hline Negative & $32(43.80)$ & 35 (48.6) & 316 (43.75) & \\
\hline Her-2 & & & & 0.000 \\
\hline Positive & $47(64.38)$ & $48(66.67)$ & $307(42.60)$ & \\
\hline Negative & $26(35.62)$ & $24(33.33)$ & $414(57.40)$ & \\
\hline Over Her-2 & & & & 0.000 \\
\hline Positive & $35(47.95)$ & $36(50.00)$ & $180(25.00)$ & \\
\hline Negative & $38(52.05)$ & $36(50.00)$ & $541(75.00)$ & \\
\hline Subtype & & & & 0.000 \\
\hline Luminal-A & $23(31.51)$ & $21(29.17)$ & $325(45.00)$ & \\
\hline Luminal-B & $26(35.62)$ & $25(34.72)$ & $162(22.50)$ & \\
\hline ERBB2+ & $21(28.77)$ & $23(31.94)$ & $144(20.00)$ & \\
\hline Basal-like & $3(4.10)$ & $3(4.17)$ & $90(12.50)$ & \\
\hline
\end{tabular}

DCIS Ductal carcinoma in situ; Family history, Family history of breast cancer in a first-degree relative; IDC Infiltrating ductal carcinoma; Mi Microinvasion; SD Standard deviation.

patients with DCIS, DCIS-Mi, and IDC groups $(P=0.51)$, but DCIS and DCIS-Mi was mainly yellow discharge, IDC was mainly bloody. The second main clinical performance was asymptomatic. Patients often see doctors because of physical examination or abnormalities in imaging (included US and mammography). There was no difference between DCIS, DCIS-Mi, and IDC groups in eczema and pain for the first clinical performances $(P<0.05)$. For diagnostic methods, the proportion of catheter winded was less in IDC than in DCIS and DCIS-Mi $(P \leq 0.001)$, while the proportion of the nodular mass was significantly more in IDC than in DCIS and DCIS-Mi by US $(P=0.003)$. There was no difference between DCIS, DCIS-Mi, and IDC in solid and cystic, structural disorder, and calcification, respectively $(P<0.05)$. For mammography feature, there was significantly difference among them in the imaging of nodular mass $(P<0.05)$, but no difference in the imaging of calcification $(P=0.431)$.

A total of 215 of those 866 patients had a history of breast operation and biopsy: 19 (26.0\%) DCIS, 20 (27.8\%) DCIS-Mi, and 176 (24.4\%) IDC. We divided the past disease into benign breast disease (BBD) and malignant breast disease (MBD) which were showed in Table 3. BBD is usually subdivided into non-proliferative lesions (fibrosis, cysts, apocrine metaplasia, fibroadenoma), 
Table 2 First clinical performances and diagnostic methods of the three patient populations: DCIS, DCIS-Mi, and IDC

\begin{tabular}{lllll}
\hline Parameter & DCIS & DCIS-Mi & IDC & $P$ value \\
\hline Total number & 73 & 72 & 721 & \\
First clinical performance & & & & \\
Nipple discharge & $9(12.3)$ & $8(11.1)$ & $63(8.7)$ & 0.51 \\
Bloody & $4(5.5)$ & $3(4.2)$ & $44(6.1)$ & 0.776 \\
Yellow & $5(6.8)$ & $5(6.9)$ & $19(3.3)$ & 0.062 \\
Palpable tumor & $48(65.8)$ & $50(69.4)$ & $518(71.8)$ & 0.52 \\
Pain & $3(4.1)$ & $5(5.9)$ & $37(5.1)$ & 0.731 \\
Eczema & $1(1.4)$ & $1(1.4)$ & $29(4.0)$ & 0.22 \\
Asymptomatic & $12(16.4)$ & $8(11.1)$ & $74(10.3)$ & 0.27 \\
Diagnostic methods & & & & \\
Ultrasound & & & & \\
Catheter widened & $65(89)$ & $56(77.8)$ & $450(62.4)$ & 0.000 \\
Solid and cystic & $3(4.1)$ & $1(1.4)$ & $21(2.9)$ & 0.589 \\
Nodular mass & $56(76.7)$ & $53(73.6)$ & $648(89.9)$ & 0.003 \\
Structural disorder & $56(76.7)$ & $44(61.6)$ & $486(67.4)$ & 0.125 \\
Calcifications & $37(50.7)$ & $34(47.2)$ & $342(47.7)$ & 0.866 \\
Mammography & & & & \\
Calcifications & $60(82.2)$ & $63(87.5)$ & $631(87.5)$ & 0.431 \\
Nodular mass & $30(41.1)$ & $25(34.7)$ & $532(73.8)$ & 0.000 \\
\hline DCIs Ductal carcinoma in situ; IDC Infiltrating ductal carcinoma; Mi & \\
Microinvasion. & & & &
\end{tabular}

proliferative lesions without atypia (lobular hyperplasia without atypia, sclerosing adenosis, papilloma), and hyperplasia with atypia (atypical ductal hyperplasia, atypical lobular hyperplasia). MBD includes IDC, invasive lobular carcinoma, invasive mucinous carcinoma, invasive tubular carcinoma, and DCIS. Then we found there was no difference between them in the past different breast benign disease types. And it was worth noting that lobular hyperplasia without atypical accounts for most. At the same time, one case of DCIS has gotten IDC in the past breast surgical or biopsy history.

\section{Discussion}

Breast cancer is the second leading cause of death due to cancer in women. In recent years, with the improvement of diagnostic methods, the detection rate of breast cancer has been increasing. DCIS accounts for approximately $10 \%$ to $15 \%$ of breast cancers detected by mammography in China $[17,18]$. Now the data suggest that carcinoma in situ can recur or progress to IDC and breast DCIS-Mi is considered to be the interim stage in the progression from DCIS to IDC $[9,10]$. The diagnosis and the ability to predict the outcome of patients with DCIS/DCIS-Mi are not satisfactory, leaded to inappropriate treatment choices. To the authors' knowledge, the hunt for molecular prognostic markers for DCIS and DCIS-Mi has not succeeded. Therefore, we urgently want to find the difference in clinicopathological, IHC, and imaging features between DCIS, DCIS-Mi, and IDC

Table 3 Past breast surgical or biopsy history of the three patient populations: DCIS, DCIS-Mi, and IDC

\begin{tabular}{|c|c|c|c|c|}
\hline Parameter & DCIS & DCIS-Mi & IDC & $P$ value \\
\hline Total number & 73 & 72 & 721 & \\
\hline Past breast surgical or biopsy history & $19(26.0)$ & $20(27.8)$ & $176(24.4)$ & 0.795 \\
\hline \multicolumn{5}{|l|}{ Benign } \\
\hline Fibrosis & $2(10.5)$ & $4(20)$ & $29(16.5)$ & 0.700 \\
\hline Cysts & $3(15.8)$ & $2(10)$ & $23(13.1)$ & 0.864 \\
\hline Apocrine metaplasia & $1(5.3)$ & $1(5.0)$ & $5(2.8)$ & 0.789 \\
\hline Fibroadenoma & $1(5.3)$ & $1(5.0)$ & $6(3.4)$ & 0.885 \\
\hline Lobular hyperplasia without atypia & $8(42.1)$ & $6(30.0)$ & $64(37.2)$ & 0.728 \\
\hline Sclerosing adenosis & $0(0)$ & $0(0)$ & $1(0.5)$ & \\
\hline Papilloma & $1(5.3)$ & $2(10.0)$ & $9(5.2)$ & 0.724 \\
\hline Atypical ductal hyperplasia & $1(5.3)$ & $2(10.0)$ & $10(5.8)$ & 0.780 \\
\hline Atypical lobular hyperplasia & $3(15.8)$ & $2(10.0)$ & $28(16.3)$ & 0.743 \\
\hline \multicolumn{5}{|l|}{ Malignant } \\
\hline \multicolumn{5}{|l|}{ IDC } \\
\hline Invasive lobular carcinoma & 0 & 0 & 0 & \\
\hline Invasive mucinous carcinoma & 0 & 0 & 0 & \\
\hline Invasive tubular carcinoma & 0 & 0 & 0 & \\
\hline DCIS & 0 & 0 & 1 & \\
\hline
\end{tabular}

DCIS Ductal carcinoma in situ; IDC Infiltrating ductal carcinoma; Mi Microinvasion. 
in order to describe the process of DCIS to IDC from the clinical aspects and discuss those results, contact treatment, and reduce the incidence.

We studied the differences of clinicopathological, IHC, and imaging features among DCIS, DCIS-Mi, and IDC. We found that there was no significant difference between patients with DCIS, DCIS-Mi, and IDC in the first symptoms in patients with previous breast history and tumor size, but there were significant different in patients' age and menopausal state. It suggested that even if patient get diseases the situations are consistent, patients with old age and menopause were more likely to have IDC. It was found that there was a significant difference in the number of patients with lymph node metastasis, and the former two were significantly less than the latter, suggesting that the latter degree of malignancy was significantly higher than former two. The first clinical performance, family history, and previous history of breast disease had no difference between DCIS, DCIS-Mi, and IDC, while the degree of malignancy in IDC was higher than in DCIS and DCIS-Mi. Except for the age and the status of menopause, we found that US and mammography played an important role in differentiating DCIS, DCIS-Mi, and IDC. US was usually routinely performed for patients because of its advantages (non-traumatic, repetitive), and it could find a mass which is $>2 \mathrm{~cm}$ [19]. This paper studied the difference of US features among DCIS, DCIS-Mi, and IDC, and found that the mass imaging of IDC patients by US were more obvious, which may be due to IDC patients' late incidence, large mass, type of intraductal carcinoma, calcification, and ill-defined infiltration. The number of DCIS and DCIS-Mi patients with catheter widened imaging by US was greater than IDC patients, which may be explained that the latter's large tumor image cover the widened catheter. Mammography played an important role in the early detection of breast cancer, especially in DCIS. According to the literature, mammography was important for detecting calcifications with high sensitivity, especially for the detection accuracy rate of DCIS. We also studied the differences among them in the mammography imaging, and found that there was no significant difference in calcification, but significant differences found in mass images. This may be due to DCIS patients' early incidence and large mass, and IDC, DCIS, and DCIS-Mi all had calcification, so mammography was not obvious, but important in the diagnosis of early DCIS with micro-calcification.

In molecular biology, the breast is a sex hormonedependent organ. Its growth, development, and cell proliferation are all influenced by estrogen and progesterone. The regulation works by estrogen receptor (ER) and progesterone receptor (PR) binding the receptors in the breast cell. ER and PR play an important role in the incidence of breast cancer; normal breast tissue of ERpositive expression will increase the risk of breast cancer [20]. ER and PR were closely related to the prognosis and endocrine therapy. The positive expression of endocrine therapy is an effective rate of $80 \%$ ER-positive tamoxifen treatment which can effectively reduce the local recurrence of DCIS [21]. A report showed that ER and PR expression range from $60 \%$ to $78 \%$ in DCIS [22]. This article basically confirmed the literature. At the same time, we found molecular markers ER and PR expression were similar in DCIS, DCIS-Mi, and IDC, which suggested hormone receptor status was determined in DCIS stage. Although there was no accurate conclusion in the breast cancer pathway, it may be a very close relationship between DCIS and IDC in the event of the development and occurrence.

Her-2 proto-oncogene product is one of the epidermal growth factor receptor (EGFR) family, with binding with ligand, changing the conformation, and causing a series of 'waterfall' types of chain reaction, then accelerating cell proliferation, accelerating cell cycle, and enhancing malignant behavior. Most studies suggested that Her-2 over-expression was related to breast cancer invasion and poor prognosis $[23,24]$. Our study found that Her-2 positive expression was $64.3 \%$ in DCIS, $66.67 \%$ in DCIS$\mathrm{Mi}$, and $42.5 \%$ in IDC. Consistent with previous reports, Her-2 express was lower in IDC than in DCIS, and was over-expressed in DCIS [25-27]. At the same time, several studies have found that normal breast tissue or benign lesions generally do not over-express HER-2 $[25,27]$. Some researches thought Her-2 positive expression was due to the process of atypical hyperplasia in DCIS, but in developing into IDC Her-2 frequently lose, or Her-2 caused the direct immune response. Another assumption was that the Her-2 negative in IDC was not developed from DCIS, but from the atypical hyperplasia $[28,29]$. According to data, we agreed with this hypothesis. So we regarded Her-2 as one of the indicators of prognosis and treatment.

We then tried to discover whether patients with DCIS, DCIS-Mi, and IDC were associated with a history of breast disease. Now due to the development of breast imaging examination, lesions could be found in the early stages. Breast disease history is helpful to evaluate the subsequent risk of breast cancer. Previous reports showed that women with proliferative breast lesions without atypia have a slightly increased risk of breast cancer, whereas women with atypical hyperplasia have a substantially increased risk [13,30-34]. In this retrospective study of breast operation history or biopsy history of patients who got DCIS, DCIS-Mi, and IDC, we found there was no difference in the proportion of previous breast operation and biopsy. We also found that DCIS, DCIS-Mi, and IDC patients whose previous medical 
history of lobular hyperplasia without atypia accounts for the large proportion may be explained by its high incidence in normal people. At the same time, hyperplasia with atypia accounts less than lobular hyperplasia without atypia, but it was accounted more than other breast disease and it also agreed with the above point of view. Finally, one case of DCIS patients recurred into IDC, indicating that DCIS has the possibility of recurrence. However, this retrospective study excluded patients with breast cancer who had not performed biopsy or operation, so it was not very accurate, and further analysis remains to be systematic prospective studies and largescale system examination.

\section{Conclusions}

In summary, through comparing DCIS, DCIS-Mi, and IDC, we found that DCIS, DCIS-Mi, and IDC were distinct entities. A larger sample size is needed for further study.

\section{Abbreviations}

BBD: Benign breast disease; DCIS: Breast ductal cancer in situ; DCIS-Mi: Breast ductal cancer in situ with microinvasion; IDC: Invasive ductal cancer; IHC: Immunohistochemical; MBD: Malignant breast disease; US: Ultrasound.

\section{Competing interests}

There are no financial or non-financial competing interests in our study.

\section{Authors' contributions}

ZW and GG-L drafted the manuscript. GE-L and ZY-L participated in the design of the study and performed the statistical analysis. ZQ and ZZ-Y conceived of the study, and participated in its design and coordination, and helped to draft the manuscript. CG-R is the main pathologist for diagnose the specimens and $\mathrm{ZH}-\mathrm{M}$ is main radiologist for evaluating the imaging of Ultrasound and Mammography. HG-L and ZX-H made up the surgical team involved in the most of patients. All authors read and approved the final manuscript.

\section{Authors' information}

Zhang Wei is a graduate of Wenzhou Medical College, Wenzhou, Zhejiang, People's Republic of China. Gao Er-Li is a graduate of Wenzhou Medical College, Wenzhou, Zhejiang, People's Republic of China. Zhou Yi-Li is a graduate of Wenzhou Medical College, Wenzhou, Zhejiang, People's Republic of China. Zhai Qi is a graduate of Wenzhou Medical College, Wenzhou, Zhejiang, People's Republic of China. Zou Zhang-Yong is a graduate of Wenzhou Medical College, Wenzhou, Zhejiang, People's Republic of China. Guo Gui-Long is Chief Physician of Department of Oncology, The First Affiliated Hospital of Wenzhou Medical College, Wenzhou, Zhejiang, People's Republic of China. Chen Guo-Rong is Chief Physician of Department of Pathology, The First Affiliated Hospital of Wenzhou Medical College, Wenzhou, Zhejiang, People's Republic of China. Zheng Hua-Min is a radiologist of Department of Radiology, The First Affiliated Hospital of Wenzhou Medical College, Wenzhou, Zhejiang, People's Republic of China. Huang Guan-li is a surgeon of department of Oncology, The First Affiliated Hospital of Wenzhou Medical College, Wenzhou, Zhejiang, People's Republic of China. Zhang Xiao-hua is Chief Physician of department of Oncology, The First Affiliated Hospital of Wenzhou Medical College, Wenzhou, Zhejiang, People's Republic of China.

\section{Acknowledgements}

The subject's financial support was provided by the department of health of Zhejiang province (No. 2009B106) and the wenzhou administration of Science \&Technology (No.Y20100008, No.Y20080081).

\section{Author details}

'Department of Oncology, The First Affiliated Hospital of Wenzhou Medical College, Wenzhou, Zhejiang, People's Republic of China. ${ }^{2}$ Department of Pathology, The First Affiliated Hospital of Wenzhou Medical College, Wenzhou, Zhejiang, People's Republic of China. ${ }^{3}$ Department of Radiology, The First Affiliated Hospital of Wenzhou Medical College, Wenzhou, Zhejiang, People's Republic of China.

Received: 8 September 2012 Accepted: 24 November 2012

Published: 8 December 2012

\section{References}

1. DeSantis C, Siegel R, Bandi P, Jemal A: Breast cancer statistics. CA Cancer J Clin 2011, 61:409-418.

2. Wiechmann L, Kuerer HM: The molecular journey from ductal carcinoma in situ to invasive breast cancer. Cancer 2008, 112:2130-2142.

3. Singletary SE, Allred C, Ashley P, Bassett LW, Berry D, Bland KI, Borgen PI, Clark G, Edge SB, Hayes DF, Hughes LL, Hutter RV, Morrow M, Page DL, Recht A, Theriault RL, Thor A, Weaver DL, Wieand HS, Greene FL: Revision of the American Joint Committee on Cancer staging system for breast cancer. J Clin Oncol 2002, 20:3628-3636.

4. Bianchi S, Vezzosi V: Microinvasive carcinoma of the breast. Pathol Oncol Res 2008, 14:105-111.

5. Schuh ME, Nemoto T, Penetrante RB, Rosner D, Dao TL: Intraductal carcinoma. Analysis of presentation, pathologic findings, and outcome of disease. Arch Surg 1986, 121:1303-1307.

6. Wong $J H$, Kopald KH, Morton DL: The impact of microinvasion on axillary node metastases and survival in patients with intraductal breast cancer. Arch Surg 1990, 125:1298-1301.

7. Strnad P: The 5th edition of the TNM classification-malignant tumors of the breast. Ceska Gynekol 1999, 64:54-57.

8. Perry N, Broeders M, de Wolf C, Tornberg S, Holland R, von Karsa L: European guidelines for quality assurance in breast cancer screening and diagnosis. Fourth edition-summary document. Ann Oncol 2008, 19:614-622.

9. de Mascarel I, MacGrogan G, Mathoulin-Pelissier S, Soubeyran I, Picot V, Coindre JM: Breast ductal carcinoma in situ with microinvasion: a definition supported by a long-term study of 1248 serially sectioned ductal carcinomas. Cancer 2002, 94:2134-2142.

10. Yu KD, Wu LM, Liu GY, Wu J, Di GH, Shen ZZ, Shao ZM: Different distribution of breast cancer subtypes in breast ductal carcinoma in situ (DCIS), DCIS with microinvasion, and DCIS with invasion component. Ann Surg Oncol 2011, 18:1342-1348.

11. Okumura Y, Yamamoto Y, Zhang Z, Toyama T, Kawasoe T, Ibusuki M, Honda Y, lyama K, Yamashita H, Iwase H: Identification of biomarkers in ductal carcinoma in situ of the breast with microinvasion. BMC Cancer 2008, $8: 287$

12. Vieira CC, Mercado CL, Cangiarella JF, Moy L, Toth HK, Guth AA: Microinvasive ductal carcinoma in situ: clinical presentation, imaging features, pathologic findings, and outcome. Eur J Radiol 2010, 73:102-107.

13. Dupont WD, Parl FF, Hartmann WH, Brinton LA, Winfield AC, Worrell JA, Schuyler PA, Plummer WD: Breast cancer risk associated with proliferative breast disease and atypical hyperplasia. Cancer 1993, 71:1258-1265.

14. Carter CL, Corle DK, Micozzi MS, Schatzkin A, Taylor PR: A prospective study of the development of breast cancer in 16,692 women with benign breast disease. Am J Epidemiol 1988, 128:467-477.

15. Nagle RB, Bocker W, Davis JR, Heid HW, Kaufmann M, Lucas DO, Jarasch ED: Characterization of breast carcinomas by two monoclonal antibodies distinguishing myoepithelial from luminal epithelial cells. J Histochem Cytochem 1986, 34:869-881.

16. Pinder SE: Ductal carcinoma in situ (DCIS): pathological features, differential diagnosis, prognostic factors and specimen evaluation. Mod Pathol 2010, Suppl 2:S8-13.

17. Yu KD, Di GH, Wu J, Lu JS, Shen KW, Shen ZZ, Shao ZM: Development and trends of surgical modalities for breast cancer in China: a review of 16year data. Ann Surg Oncol 2007, 14:2502-2509.

18. Fan L, Zheng Y, Yu KD, Liu GY, Wu J, Lu JS, Shen KW, Shen ZZ, Shao ZM: Breast cancer in a transitional society over 18 years: trends and present status in Shanghai. China. Breast Cancer Res Treat 2009, 117:409-416.

19. Fu LN, Wang Y, Huang YH: Value of ultrasound elastography in detecting small breast tumors. Chin Med J (Engl) 2011, 124:2384-2386. 
20. Lawson JS, Field AS, Tran DD, Killeen J, Maskarenic G, Ishikura H, Trichopoulos D: Breast cancer incidence and estrogen receptor alpha in normal mammary tissue-an epidemiologic study among Japanese women in Japan and Hawaii. Int J Cancer 2002, 97:685-687.

21. Collins LC, Schnitt SJ: HER2 protein overexpression in estrogen receptorpositive ductal carcinoma in situ of the breast: frequency and implications for tamoxifen therapy. Mod Pathol 2005, 18:615-620.

22. Daly MB: Tamoxifen in ductal carcinoma in situ. Semin Oncol 2006, 33:647-649.

23. Ooi A, Takehana T, Li X, Suzuki S, Kunitomo K, lino H, Fujii H, Takeda Y, Dobashi Y: Protein overexpression and gene amplification of HER-2 and EGFR in colorectal cancers: an immunohistochemical and fluorescent in situ hybridization study. Mod Pathol 2004, 17:895-904.

24. Pellikainen JM, Ropponen KM, Kataja W, Kellokoski JK, Eskelinen MJ, Kosma VM: Expression of matrix metalloproteinase (MMP)-2 and MMP-9 in breast cancer with a special reference to activator protein-2, HER2, and prognosis. Clin Cancer Res 2004, 10:7621-7628.

25. Schimmelpenning H, Eriksson ET, Falkmer UG, Azavedo E, Svane G, Auer GU: Expression of the c-erbB-2 proto-oncogene product and nuclear DNA content in benign and malignant human breast parenchyma. Virchows Arch A Pathol Anat Histopathol 1992, 420:433-440.

26. Pavelic ZP, Pavelic L, Lower EE, Gapany M, Gapany S, Barker EA: Preisler HD: c-myc, c-erbB-2, and Ki-67 expression in normal breast tissue and in invasive and noninvasive breast carcinoma. Cancer Res 1992, 52:2597-2602.

27. Allred DC, Clark GM, Molina R, Tandon AK, Schnitt SJ, Gilchrist KW, Osborne CK, Tormey DC, McGuire WL: Overexpression of HER-2/neu and its relationship with other prognostic factors change during the progression of in situ to invasive breast cancer. Hum Pathol 1992, 23:974-979.

28. Hoque A, Sneige N, Sahin AA, Menter DG, Bacus JW, Hortobagyi GN, Lippman SM: Her-2/neu gene amplification in ductal carcinoma in situ of the breast. Cancer Epidemiol Biomarkers Prev 2002, 11:587-590.

29. Mylonas I, Makovitzky J, Jeschke U, Briese V, Friese K, Gerber B: Expression of Her2/neu, steroid receptors (ER and PR), Ki67 and p53 in invasive mammary ductal carcinoma associated with ductal carcinoma in situ (DCIS) versus invasive breast cancer alone. Anticancer Res 2005, 25:1719-1723

30. Dupont WD, Page DL: Risk factors for breast cancer in women with proliferative breast disease. N Engl J Med 1985, 312:146-151.

31. London SJ, Connolly JL, Schnitt SJ, Colditz GA: A prospective study of benign breast disease and the risk of breast cancer. JAMA 1992, 267:941-944.

32. Hartmann LC, Sellers TA, Frost MH, Lingle WL, Degnim AC, Ghosh K Vierkant RA, Maloney SD, Pankratz VS, Hillman DW, Suman VJ, Johnson J, Blake C, Tlsty T, Vachon CM, Melton $\sqcup$ 3rd, Visccher DW: Benign breast disease and the risk of breast cancer. N Engl J Med 2005, 353:229-237.

33. Worsham MJ, Raju U, Lu M, Kapke A, Botttrell A, Cheng J, Shah V, Savera A Wolman SR: Risk factors for breast cancer from benign breast disease in a diverse population. Breast Cancer Res Treat 2009, 118:1-7.

34. Kabat GC, Jones JG, Olson N, Negassa A, Duggan C, Ginsberg M, Kandel RA, Glass AG, Rohan TE: A multi-center prospective cohort study of benign breast disease and risk of subsequent breast cancer. Cancer Causes Control 2010, 21:821-828.

\section{Submit your next manuscript to BioMed Central and take full advantage of:}

- Convenient online submission

- Thorough peer review

- No space constraints or color figure charges

- Immediate publication on acceptance

- Inclusion in PubMed, CAS, Scopus and Google Scholar

- Research which is freely available for redistribution

Submit your manuscript at www.biomedcentral.com/submit
Ciomed Central 